\title{
Gait ataxia as a presenting symptom of malignant epidural spinal cord compression
}

\author{
S.J. Karp and R.T.K. Ho
}

Meyerstein Institute of Radiotherapy and Oncology and Department of Neurology, Middlesex Hospital, Mortimer Street, London W1, UK.

\begin{abstract}
Summary: A 68 year old man with prostatic carcinoma developed spinal cord compression. The sole presenting feature was painless gait ataxia indistinguishable from cerebellar ataxia. He was investigated for cerebellar disease but the correct diagnosis was made when he subsequently presented with paraplegia and classical signs of spinal cord compression. Surgical decompression failed to produce neurological recovery.

We discuss the importance of recognizing this unusual presentation of spinal cord compression, its possible mechanism and evidence that it may be under-reported.
\end{abstract}

\section{Introduction}

The symptoms and signs of spinal cord compression are well known (Walton, 1977a; Henson \& Urich, 1982). However, presentation with unusual features may have serious consequences as the following report, on a patient who presented solely with a gait ataxia, illustrates.

\section{Case report}

A 68 year old man presented in April 1983 with a 4 year history of prostatism. A retropubic prostatectomy was performed. Histology showed a poorly differentiated adenocarcinoma of the prostate, and he was commenced on stilboestrol. Sixteen months later prostatic symptoms returned and in September 1984 he was admitted with acute urinary retention. He was found to have a locally advanced carcinoma of the prostate, and a suprapubic catheter was inserted. A transurethral resection of the prostate and subcapsular orchidectomy were performed and he was subsequently referred for radiotherapy.

$A^{99 m}$ Tc bone scan, performed for staging purposes, showed intense focal abnormal uptake involving the right anterior 3rd and 5th ribs and the body of D5 vertebra (Figure 1).

A palliative dose of irradiation was given to the prostate. Towards the end of the course of radiotherapy he became aware of mild discomfort in his dorsal spine at about the level of D5. Two days later he

Correspondence: S.J. Karp, B.Sc., M.R.C.P.

Accepted: 3 February 1986

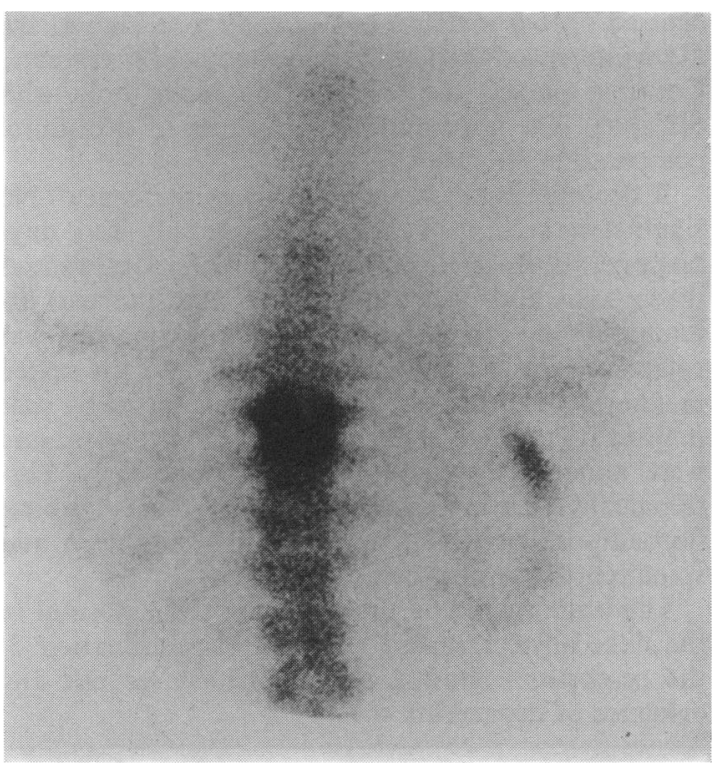

Figure 1 Technetium $99^{\mathrm{m}}$ bone scan showing marked focal increased uptake in D6 vertebral body.

reported that over the preceding week he had felt 'wobbly on his legs'. Neurological examination revealed a slightly ataxic gait but no other abnormality; in particular there were no signs of spinal cord compression. X-rays of the dorsal spine showed 
sclerosis of D6 vertebral body and loss of definition of the left pedicle.

Over the next 2 days his gait became more ataxic and an urgent neurological opinion was sought. He was found to have a severely ataxic gait and some impairment of heel-shin testing bilaterally. There was no evidence of an upper motor neurone lesion or a sensory level and joint position sense was entirely normal. It was felt that the most likely diagnosis was of a cerebellar metastasis or a non-metastatic cerebellar syndrome. No signs of spinal cord compression were found and computed tomographic head scanning was performed and proved normal.

One week later the patient was admitted as an emergency with a 12 hour history of severe mid-dorsal back pain radiating in a band-like fashion around his chest, and inability to walk or pass urine. Physical examination revealed an enlarged bladder. There was bilateral spasticity in both legs with power reduced to MRC 3/5. Reflexes were symmetrical and brisk and both plantar responses were extensor. Pain sensation was impaired in the legs and lower trunk and there was a sensory level to pin-prick at D6. Myelography showed substantial narrowing of the spinal theca centred on D6 vertebra but extending as high as the D5/6 interspace and as low as the body of D7 vertebra. Contrast passed the level of the block only with difficulty. The appearances were those of extradural compression (Figure 2).

A decompressive laminectomy was performed. No significant return of function occurred. Ten days postoperatively he developed a left iliofemoral venous thrombosis and died 2 days later. Post-mortem examination showed that the vertebral bone marrow was extensively replaced by pale tumour, and the spinal meninges were thickened to $0.5 \mathrm{~cm}$ diameter by pale infiltrating tumour in the region of D6 vertebra, and were adherent to the spinal cord posteriorly. Histologically, there was infiltration of the extradural fibroadipose tissue but infiltration of the dura and spinal cord was not seen.

The brain including the cerebellum was normal to the naked eye. Detailed histological examination of the cerebellum showed neither metastases nor any evidence of degeneration.

\section{Discussion}

The clinical picture of epidural spinal cord compression is usually fairly uniform (Henson \& Urich, 1982). Local or radicular pain occurs in up to $96 \%$ of patients (Chade, 1976; Gilbert et al., 1978; Henson \& Urich, 1982 ) and this is usually the earliest symptom. Increasing difficulty with walking develops, often in the absence of objective neurological signs, and progresses at a variable rate to a spastic paraplegia with increased

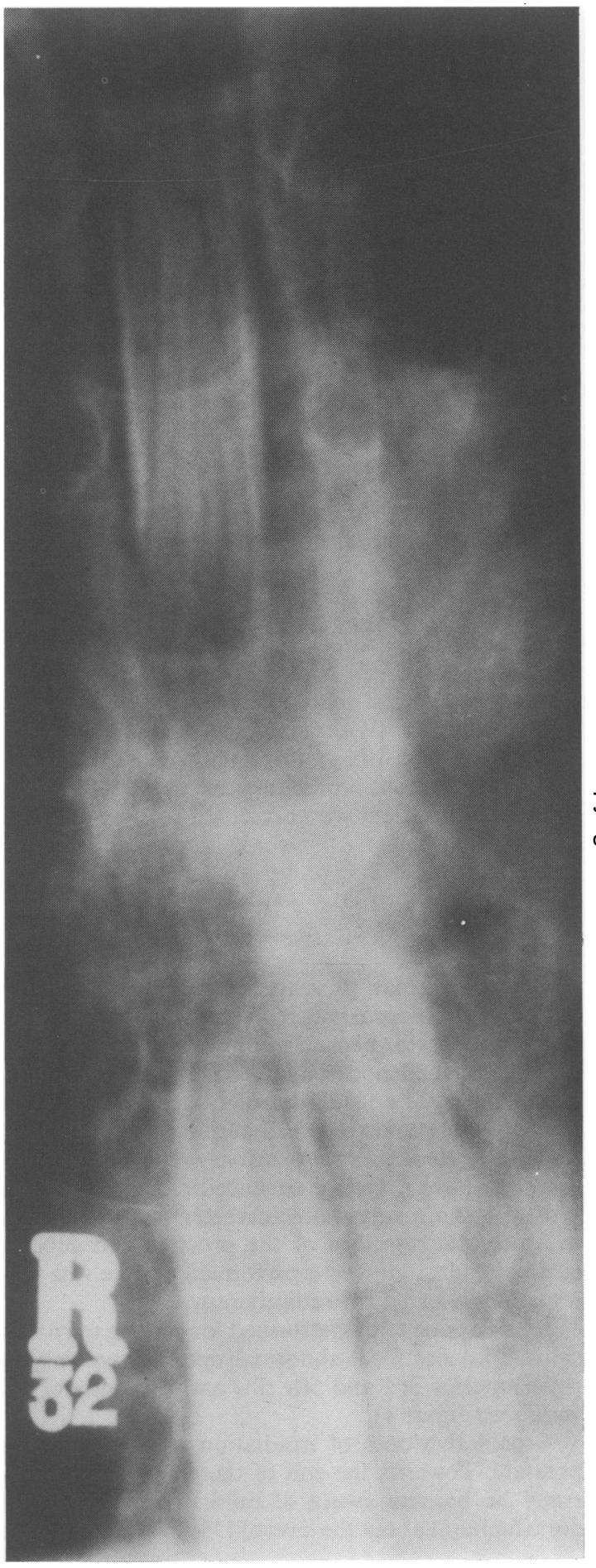

Figure 2 AP myelogram demonstrating spinal cord compression at the level of D6 vertebra. 
muscle tone, brisk reflexes and extensor plantar responses. Sensory loss is usually present although a distinct level occurs in only one third of patients. An acute, rapidly progressive syndrome may occur, especially following vertebral collapse. Herpes zoster at the level of root involvement and flexor spasms are less common manifestations (Chade, 1976; Gilbert $\boldsymbol{e t}$ al., 1978; Henson \& Urich, 1982).

Ataxia as an isolated presenting symptom is apparently extremely uncommon. Standard textbook descriptions and several large reported series of malignant epidural spinal cord compression make no mention of it (Paillas et al., 1976; Chade et al., 1976; Walton, 1977a; Stark et al., 1982; Henson \& Urich, 1982; Tang et al., 1983). Gilbert et al. (1978) in a review of 135 cases noted ataxia without pain or motor or sensory loss as the presenting complaint in two patients and commented that in seven other patients severe gait ataxia was the most striking sign on examination. In these patients they found that proprioception was either normal or only mildly impaired and, in isolation could not account for the gait difficulties. One of their patients, like the present case, was investigated for cerebellar disease before spinal cord compression could be recognized as the causative factor.

A subsequent series from the same centre (Greenberg et al., 1980) reported on 83 patients, 6 of whom had ataxia at diagnosis. One patient had ataxia as an initial symptom but whether this was the sole presenting feature is not stated.

The cause of the ataxia is not known. However, anatomically it is known that the anterior and pos- terior spinocerebellar tracts convey proprioceptive information from muscles and tendons to the cerebellum and it has, therefore, been suggested that involvement of these spinal pathways might produce ataxia without gross loss of postural sensibility (Walton, 1977b).

Radiotherapy to skeletal metastases may not be indicated despite positive bone scintigraphy or radiology. Even in patients with malignancy, back pain may have a benign cause in up to one third of patients (Galasko et al., 1982). However, if impending spinal cord compression had been thought likely, urgent radiotherapy with systemic steroid cover would almost certainly have been initiated in the hope of preventing further deterioration and reversing the ataxia. Following prompt management of spinal cord compression, up to $36 \%$ of patients will be alive after one year and $50 \%$ of survivors can expect to be able to walk at one year (Gilbert et al., 1978; Starke et al., 1982; Henson \& Urich, 1982).

We would, therefore, suggest that in patients who develop a gait ataxia with negative intracranial investigations, and especially in the presence of known vertebral metastasis, the diagnosis of spinal cord compression is seriously considered so that appropriate investigations are initiated at an early stage.

\section{Acknowledgements}

We would like to thank Professor R.J. Berry for permission to report this case, the Department of Medical Illustration, Middlesex Hospital, for preparing the photographs, and Miss Julia Lintott for typing the manuscript.

\section{References}

CHADE, H.O. (1976). Metastatic tumours of the spine and spinal cord. In Handbook of Clinical Neurology, Vinken, P.J. and Bruyn, G.W. (eds). Vol. 20, pp. 415-433. North Holland: Amsterdam.

GALASKO, C.S.B., BUSH, H. \& SUTTON, H.L. (1982). Bone, cartilage and synovium. In Treatment of Cancer, Halnan, K.E. (ed). pp. 623-651. Chapman and Hall: London.

GILBERT, R.W., KIM, J.H. \& POSNER, J.B. (1978). Epidural spinal cord compression from metastatic tumour: diagnosis and treatment. Annals of Neurology, 3, 40.

GREENBERG, H.S., KIM, J.H. \& POSNER, J.B. (1980). Epidural spinal cord compression from metastatic tumor: results with a new treatment protocol. Annals of Neurology, 8, 361 .

HENSON, R.A. \& URICH, H. (1982). In Cancer and the Nervous System: the Neurological Manifestations of Systemic Malignant Disease, pp. 120-154. Blackwell Scientific Publications: Oxford, London.

PAillas, J.E., ALlieZ, B. \& PELlet, W. (1976). Primary and secondary tumours of the spine. In Handbook of Clinical Neurology, Vinken, P.J. and Bruyn, G.W. (eds). Vol. 20, pp. 19-54. North Holland: Amsterdam.

STARK, R.J., HENSON, R.A. \& EVANS, S.J.W. (1982). Spinal metastases. A retrospective survey from a general hospital. Brain, 105, 189.

TANG, S.F., BYFIELD, S.J., SHARP, T.R., UTLEY, F.J., QUINEL, L. \& SEAGREN, S.L. (1983). Prognostic factors in the management of metastatic epidural spinal cord compression. Journal of Neuro-Oncology, 1, 21.

WALTON, J.N. (1977a). Disorders of the spinal cord and cauda equina. In Brain's Diseases of the Nervous System, 8th edition. pp.715-786. Oxford University Press: Oxford.

WALTON, J.N. (1977b). In Brain's Diseases of the Nervous System, 8th edition. pp. 43-44. Oxford University Press: Oxford, London. 